\title{
Human Equilibrative Nucleoside Transporter 1 (hENT1) Levels Predict Response to Gemcitabine in Patients With Biliary Tract Cancer (BTC)
}

\author{
D. Santini ${ }^{*}, 1$, G. Schiavon ${ }^{1}$, B. Vincenzi ${ }^{1}$, C.E. Cass $^{2}$, E. Vasile ${ }^{3}$, A.D. Manazza ${ }^{4}$, V. Catalano ${ }^{5}$,

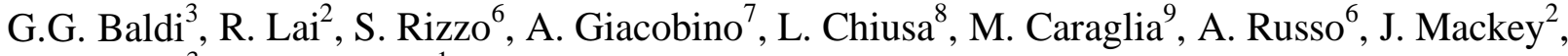 \\ A. Falcone ${ }^{3}$ and G. Tonini ${ }^{1}$
}

\begin{abstract}
${ }^{I}$ Medical Oncology, University Campus Bio-Medico, Rome, Italy; ${ }^{2}$ Cross Cancer Institute and Department of Oncology, University of Alberta, Edmonton, Alberta, Canada; ${ }^{3}$ Unit of Medical Oncology 2, Azienda-Ospedaliero Universitaria Pisana, Istituto Toscano Tumori and Department of Oncology, Transplantes and New Technologies in Medicine, University of Pisa, Italy; ${ }^{4}$ CeRMS, Molecular Oncology, University of Torino, Torino, Italy; ${ }^{5}$ Department of Medical Oncology, Azienda Ospedaliera “Ospedale San Salvatore”, Pesaro, Italy; ${ }^{6}$ Department of Surgical and Oncological Sciences, Università degli Studi di Palermo, Palermo, Italy; ${ }^{7}$ Department of Clinical Oncology, San Giovanni Battista Hospital, Torino, Italy; ${ }^{8}$ Department of Biomedical Sciences and Human Oncology, University of Torino, Torino, Italy; ${ }^{9}$ Department of Biochemistry and Biophysics Second University of Naples, Naples, Italy
\end{abstract}

\begin{abstract}
Background and aim: Translational data suggest that nucleoside transporters, in particular human equilibrative nucleoside transporter 1 (hENT1), play an important role in predicting clinical outcome after gemcitabine chemotherapy for several types of cancer. The aim of this study was to retrospectively determine patients' outcome according to the expression of hENT1 in tumoral cells of patients receiving gemcitabine-based therapy.

Materials and Methods: The immunohistochemistry analysis was performed on samples from thirty-one patients with unresectable biliary tract cancer (BTC) consecutively treated with first line gemcitabine-based regimens.

Results: Positive hENT1 staining patients were 21 (67.7\%); negative hENT1 staining patients were 10 (32.3\%). Statistical analysis revealed no association between baseline characteristics, toxicities and tumor response to gemcitabine and hENT1 levels. In the univariate analysis, HENT1 expression was significantly correlated with time to progression (TTP) ( $\mathrm{p}=0.0394$; HR 2.902, 95\%CI 1.053-7.996). The median TTP was 6.33 versus 2.83 months, respectively in patients with positive versus negative hENT1 staining. Moreover, patients with positive hENT1 expression showed a longer median overall survival when compared with patients with low hENT1 expression (14 versus 7 months, respectively), but this difference did not reach the statistical significance $(\mathrm{p}=0.128)$.

Conclusions: Therefore, hENT1 may be a relevant predictive marker of benefit from gemcitabine-based therapies in patients with advanced BTC.
\end{abstract}

Keywords: Biliary tract cancer, gemcitabine, hENT1, predictive factor.

\section{INTRODUCTION}

Biliary tract cancers (BTCs) are rare tumors and represent a major challenge for surgical, medical, and radiation oncologists. Unfortunately, the majority of these tumours are not resectable at the time of initial diagnosis, and patients with advanced disease show a poor prognosis. The efficacy of conventional cytotoxic agents (e.g., 5-fluorouracil, mitomycin-C, and cisplatin) seems to be low, and currently there is no agreement about the optimal chemotherapeutic regimen in this setting [1].

The nucleoside pyrimidine analogue gemcitabine is the most effective single agent in advanced BTCs, where it has been shown to improve clinical symptoms and to modestly extend survival [2]. Gemcitabine appears to be a reasonable alternative to the best supportive care in this setting, on the basis of encouraging results in non-randomized clinical studies [2,3]. For the first time, at the 2009 American Soci-

*Address correspondence to this author at the Department of Medical Oncology, University Campus Bio-Medico, Via Alvaro del Portillo, 20000128 Roma, Italy; Tel: + 39-06-225411242; Fax: +39-06-225411936;

E-mail: d.santini@unicampus.it ety of Clinical Oncology Annual meeting, the final results of a phase III randomized trial comparing gemcitabine with or without cisplatin have been presented. This study showed a statistical significant advantage of the gemcitabine-based doublet in terms of progression free survival (PFS) and median survival ${ }^{1}$. Consequently, chemotherapy with a combination of gemcitabine and cisplatin represents the new standard of therapy in advanced BTCs. Because the biochemical targets of gemcitabine are intracellular, the translocation through the plasma membrane is a necessary step for the drug to exert its cytotoxic effect. Gemcitabine is a hydrophilic molecule and does not cross the plasma membrane by diffusion. In fact, an efficient cellular uptake requires the presence of specialized plasma membrane nucleoside transporter (NT) proteins [4]. The human equilibrative nucleoside transporter 1 (hENT1) is a ubiquitous protein and is the major transporter by which gemcitabine enters cultured human cells and hematopoietic progenitor cells [5]. The abundance and distribution of hENT1 protein can be evaluated using immunohistochemistry, and it has been already assessed in a

${ }^{1}$ Furuse, J.; Okusaka, T.; Miyazaki, M.; Taniai, H.; Nimura, Y. A randomized study of gemcitabine/cisplatin versus single-agent gemcitabine in patients with biliary tract cancer. J. Clin. Oncol. 2009, 27(15s), Abstr 4579. 
number of malignant and benign tissues [6-9]. Interestingly, immunohistochemical (IHC) variations of hENT1 in pancreatic adenocarcinoma samples significantly correlate with median survival after gemcitabine therapy in patients with advanced disease [10]. Moreover, IHC analysis of hENT1 has recently been shown to be predictive of benefit from gemcitabine chemotherapy in resected pancreatic cancers [11].

There are limited data about hENT1 in BTCs. Studies involving BTC cells (OCUG-1 and HuCTT1) have indicated that hENT1 is overexpressed and it is the major gemcitabine transporter, representing the most important determinant of gemcitabine sensitivity [12]. Our research group has recently demonstrated that hENT1 is expressed in about $30 \%$ of ampullary cancers and its expression provides prognostic information in this type of patients [13].

The role of hENT1 expression in predicting the activity of a gemcitabine-based chemotherapy in advanced BTCs is lacking in literature.

Considering this scenario, we studied the expression of hENT1 by IHC analysis in tumour samples from 31 BTC patients receiving a gemcitabine-based therapy and we correlated these biological results with clinical outcome.

\section{MATERIALS AND METHODS}

\section{Clinical Data and Tumour Specimen Acquisition}

The analysis was performed on samples from 31 patients with unresectable locally advanced or metastatic BTC con- secutively treated with gemcitabine-based regimens between June 2001 and June 2008 in four Italian oncological centers. Adequate tumour biopsy specimens obtained before starting a gemcitabine-based chemotherapy were available for all the patients. Permission to perform this study was obtained by the local ethical board. The histopathological classification was based on WHO guidelines. Clinical staging was based on an initial evaluation consisting of a clinical assessment, computed tomography (CT) of the chest and abdomen and magnetic resonance imaging (MRI) of the abdomen. TNM classification (as reported in the ESMO Clinical Recommendation for diagnosis, treatment and follow-up) was used for the clinical staging [14]. The clinicopathological characteristics of all the patients are listed in Table $\mathbf{1}$. The patient population included 18 males and 13 females, with a median age of 70 (range, $52-83$ years).

All specimens underwent a gross anatomical examination according to the procedure described by Rosai [15], including the evaluation of all the anatomical structures. Response was evaluated in patients who had received at least two or more courses of chemotherapy, using standard response criteria. Response evaluation has been performed after two or three cycles (6-9 weeks) of chemotherapy by clinical evaluation, blood tests and by repeating the initially abnormal radiologic examinations [14]. Complete response was defined as the disappearance of all signs of disease both at clinical and radiological examination. Partial response was defined by a reduction of more than $50 \%$ in the sum of products of the largest perpendicular diameters of all tumour localizations, with absence of new tumour lesions. Stable disease was defined as a reduction of less than $50 \%$ or an increase less than

Table 1. Patients' Characteristics

\begin{tabular}{|c|c|c|}
\hline & & \# tot Patients (\%) \\
\hline \hline Gender & & 31 \\
& $\mathrm{M}$ & $18(58.1)$ \\
& $\mathrm{F}$ & $13(41.9)$ \\
\hline Performance status (ECOG) & 0 & $25(80.6)$ \\
& 1 & $6(19.4)$ \\
\hline Age (ys) & Median (Range) & $70(50-86)$ \\
& $<65$ & $12(38.7)$ \\
& $\geq 65$ & $19(61.3)$ \\
\hline Primary Tumor location & Gall bladder & $9(29)$ \\
& Ampulla & $5(16.1)$ \\
& Intraepatic biliary tract & $4(12.9)$ \\
& Extraepatic biliary tract & $13(42)$ \\
\hline Chemotherapy & Gem + Capecitabine & $18(58.1)$ \\
& Gem + Oxaliplatin & $4(12.9)$ \\
& Gem + Fluorouracil & $1(3.2)$ \\
& Gem alone & $8(25.8)$ \\
\hline TTP (months) & Median & $4.83 \mathrm{~ms}$ \\
\hline OS (months) & Median & $13.33 \mathrm{~ms}$ \\
\hline
\end{tabular}

F: female, Gem: gemcitabine, M: male, ms: months, OS: overall survival, TTP: time to progression. 
$25 \%$ of tumour lesions. Tumour progression was defined as an increase more than $25 \%$ in the size of tumour lesions or the appearance of a new lesion.

\section{Tissue Preparation and Immunostaining}

Representative tumour blocks were sectioned at $3 \mu \mathrm{m}$ thickness for IHC studies. The sections were deparaffinized with three immersions in xylene baths (10 minutes each) followed by serial washes in graded alcohol from 100 to $50 \%$. After rinsing in water, slides were placed in $250 \mathrm{~mL}$ of high $\mathrm{pH} 1 \mathrm{X}$ DAKO target antigen retrieval solution and microwaved in TT-mega Milestone (ESBE Scientific, Markham, Ontario, Canada) under controlled temperature and high pressure for 10 minutes at $100^{\circ} \mathrm{C}$. After cooling in water for 6 minutes, slides were rinsed with water and peroxidase blocked in $3 \%$ hydrogen peroxide solution with methanol for 10 minutes then washed in running water for 10 minutes. PBS ( $\mathrm{pH}$ 7.2) was used for rinsing before incubation in a humidified chamber overnight at $4{ }^{\circ} \mathrm{C}$ with appropriate dilutions of anti-hENT1 mouse monoclonal antibody developed and characterized as described previously [13]. Then, sections were rinsed with PBS, immersed in buffer for 5 minutes, incubated with goat anti-mouse dextran conjugate (DAKO Envision) for 30 minutes, followed by soaking in PBS. DAKO diaminobenzidine liquid chromagen was placed on the samples for 5 minutes, then rinsed, after which the slides were soaked in $1 \%$ CuSO4 for 5 minutes. Subsequently, the sections were rinsed, counterstained with hematoxylin, dehydrated through graded alcohol and xylene, and finally coverslipped. Negative controls were provided by omitting the primary antibody. Immunostaining was assessed by two independent pathologists blinded to clinical characteristics and outcomes.

Scoring for hENT1 was based on relative intensities of staining of the biliary tract tumor with reference to the normally present hENT1 staining of cell membranes of lymphocytes. These internal references were then used as internal positive controls between slides and samples as well as for the staining procedure. Biliary tract tumor tissue was then evaluated by comparison with the internal controls.

A three-point scale has been used for qualitatively scoring the intensity of staining, where 0 (absent or negative) indicates no staining, 1+ (weak) indicates positive staining but less intense than or equal to the positive internal control and $2+$ (strong) indicates positive staining more intense than the positive internal control.

Samples with regions of varying staining intensities of hENT1 were scored and the percentages of each staining intensity were recorded (Fig. 1).

The final hENT1 score for each sample was negative (in case of "score 0 " $\geq 50 \%$ of cells) or positive (in case of "score 0 " $<50 \%$ of cells and any percentage of "score $1+$ " and/or "score 2+").

\section{Statistical Analysis}

The following clinical characteristics and variables were considered and so categorized: age $(<65$ vs $\geq 65)$, gender

\section{hENT 1 immunostaining}

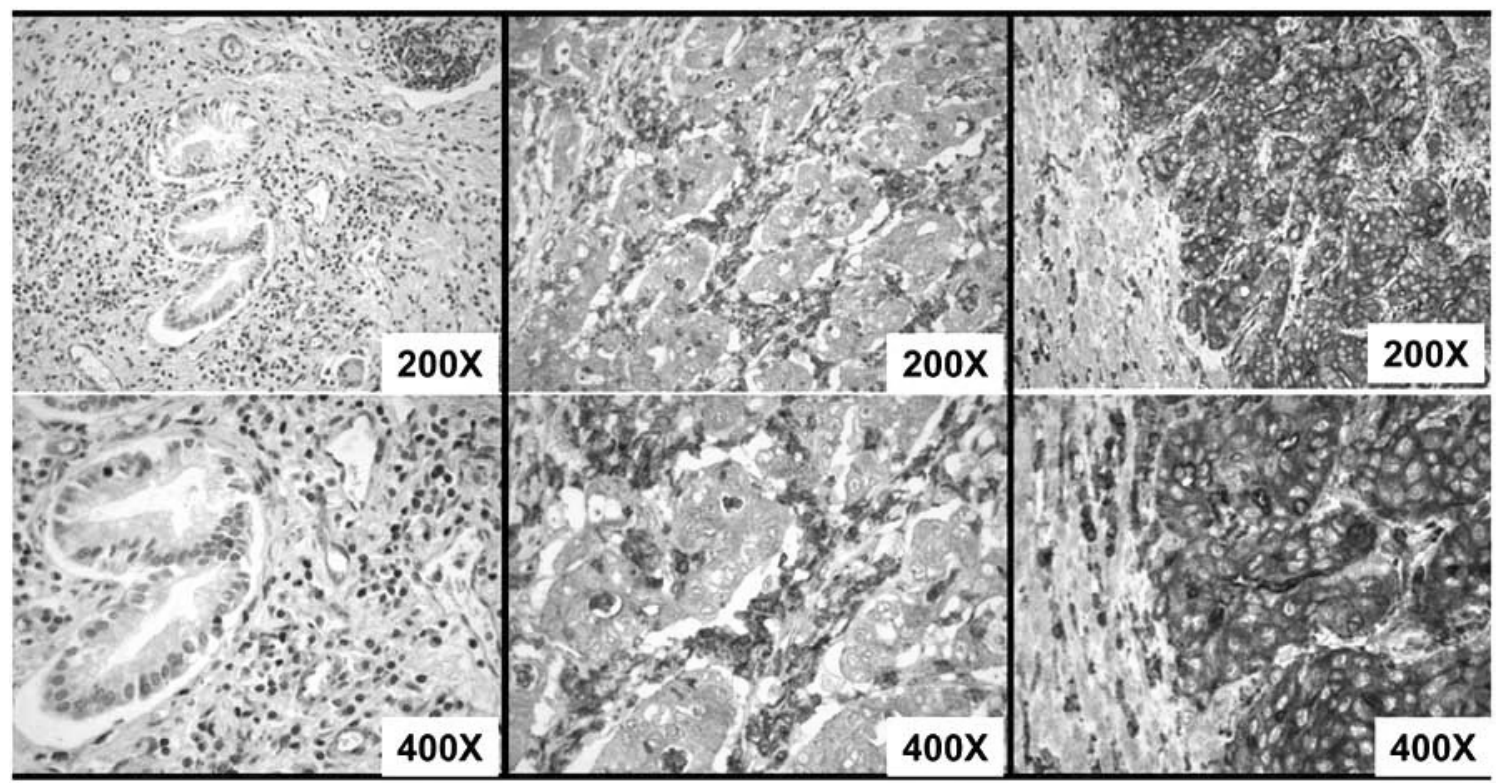

$\begin{array}{llll}\text { Score: } & 0 & 1+ & 2+\end{array}$

Fig. (1). Illustrations for the hENT1 staining. The positive staining in the infiltrating lymphocytes served as a positive internal control. A three-point scale has been used for qualitatively scoring the intensity of staining, where 0 (absent or negative) indicates no staining, $1+$ (weak) indicates positive staining but less intense than or equal to the positive internal control and $2+$ (strong) indicates positive staining more intense than the positive internal control. 
(male vs female), performance status (ECOG 0 vs 1) and primary tumor location (gall bladder vs ampulla vs intrahepatic biliary tract vs extrahepatic biliary tract). Clinical response after three cycles of chemotherapy has been evaluated (progressive disease versus stable disease versus partial response). Regarding toxicities, the worst hematologic and non-hematologic have been also dichotomized (grades 1, 2 vs grade 3 or higher). tive.

hENT1 expression was dichotomized as negative vs posi-

Fisher's exact test and Chi-square test for trend were applied to assess the association between hENT1 expression and the above mentioned baseline clinical characteristics and variables.

Time to progression (TTP) was considered as the period of time from the starting date of the treatment to the first observation of disease progression. The overall survival (OS) time was calculated as the period of time from the starting date of the treatment until death for any cause or until the last follow-up. Univariate analysis for correlation between survival (TTP and OS) and both clinical variables and hENT1 expression, was estimated by the Kaplan-Meier method and groups were compared by the log-rank test. Moreover, results were expressed as Hazard Ratio (HR).

$P$ values $<0.05$ were considered statistically significant in two-tailed tests. Prism 5 (GraphPad Software, Inc.) was used to perform the statistical analysis.

\section{RESULTS}

\section{Patient Population}

Primary sites were so distributed: intrahepatic biliary tract in 4 patients $(12.9 \%)$; extrahepatic biliary tract in 13 patients (42\%); gall bladder in 9 patients (29\%); ampulla in 5 patients $(16.1 \%)$. All the patients were treated with gemcitabine, either alone or in combination, mostly with capecitabine or oxaliplatin, and less often with fluorouracil. The median of cycles received was 11 (range 3-20). The median survival from initiation of gemcitabine chemotherapy was 13.33 months and the median TTP was 4.83 months.

After obtaining informed consent in accordance with institutional guidelines, all the patients underwent a tumor biopsy followed by chemotherapy.

Table 2. Correlations Between Immunohistochemical Data and Clinical, Pathological and Therapeutic Data

\begin{tabular}{|c|c|c|c|c|}
\hline & & hENT1 neg & hENT1 pos & p value \\
\hline \# of patients (\%) & & $10(32.3)$ & $21(67.7)$ & \\
\hline \multirow[t]{2}{*}{ Gender } & M & 6 & 12 & \multirow[t]{2}{*}{$\mathrm{ns}^{*}$} \\
\hline & $\mathrm{F}$ & 4 & 9 & \\
\hline Performance status & 0 & 4 & 11 & $\mathrm{~ns}^{*}$ \\
\hline (ECOG) & 1 & 0 & 3 & \\
\hline \multirow[t]{3}{*}{ Age (ys) } & Median (Range) & 67 & 71 & \multirow{3}{*}{ ns* } \\
\hline & $<65$ & 5 & 7 & \\
\hline & $\geq 65$ & 5 & 14 & \\
\hline \multirow[t]{4}{*}{ Primary Tumor location } & Gall bladder & 2 & 7 & \multirow[t]{4}{*}{$0.4138 * *$} \\
\hline & Ampulla & 2 & 3 & \\
\hline & Intrahepatic biliary tract & 0 & 4 & \\
\hline & Extrahepatic biliary tract & 6 & 7 & \\
\hline \multirow{3}{*}{$\begin{array}{l}\text { Clinical Response } \\
\left(3^{\text {rd }} \text { cycle of CTX }\right)\end{array}$} & $\mathrm{PD}$ & 4 & 8 & \multirow[t]{3}{*}{$0.7353^{* *}$} \\
\hline & SD & 1 & 5 & \\
\hline & PR & 3 & 8 & \\
\hline \multirow{2}{*}{$\begin{array}{c}\text { Non-hematologic toxicity } \\
\text { (WHO) }\end{array}$} & G0-G2 & 2 & 12 & \multirow[t]{2}{*}{$\mathrm{ns}^{*}$} \\
\hline & G3-G4 & 2 & 2 & \\
\hline Hematologic toxicity & G0-G2 & 4 & 11 & $\mathrm{~ns}^{*}$ \\
\hline (WHO) & G3-G4 & 0 & 3 & \\
\hline \multirow[t]{2}{*}{ TTP (months) } & Median & \multirow[t]{2}{*}{$2.83 \mathrm{~ms}$} & \multirow[t]{2}{*}{$6.33 \mathrm{~ms}$} & $0.03(\operatorname{HR} 2.9)^{* * *}$ \\
\hline & $95 \% \mathrm{CI}$ & & & $1.05-7.99$ \\
\hline \multirow[t]{2}{*}{ OS (months) } & Median & \multirow[t]{2}{*}{$7 \mathrm{~ms}$} & \multirow[t]{2}{*}{$14 \mathrm{~ms}$} & $0.12(\operatorname{HR} 2.08)^{* * *}$ \\
\hline & $95 \% \mathrm{CI}$ & & & $0.8-5.34$ \\
\hline
\end{tabular}

CTX: chemotherapy, F: female, M: male, ms: months, ns: not significant, OS: overall survival, PD: progressive disease, PR: partial response, SD: stable disease, TTP: time to progression, ys: years.

*Fisher's exact test, ** Chi-square test for trend, ***Log-rank (Mantel-Cox) Test 


\section{Immunohistochemical Data}

Tumor samples of all 31 patients have been evaluated for hENT1 immunohistochemistry. The localization of the hENT1 immunostaining was predominantly at plasma membrane, although a cytoplasmic staining was occasionally observed. Positive hENT1 staining patients were $21(67.7 \%)$, negative hENT1 staining patients were 10 (32.3\%). Distribution of hENT1 immunostainig in different histotypes (positive/negative) was: gallbladder (7/2); ampulla (3/2); intrahepatic BTC (4/0); extrahepatic BTC (7/6).

\section{Correlations Between Immunohistochemical Data and Clinical Outcome}

To study a possible correlation between immunohistochemical data and patient outcome, tumor samples were classified as positive or negative for hENT1.

At the time of analysis (June 2009), after a median follow-up of 13 months, $25(80.6 \%)$ patients were dead and 6 $(19.4 \%)$ patients were alive. Overall, $28(90.3 \%)$ patients progressed under a gemcitabine-based chemotherapy and only $3(9.7 \%)$ patients, at the time of the statistical analysis, were on therapy with gemcitabine.

There was not statistical association between baseline characteristics (age, gender, ECOG performance status and site of primary tumor) and hENT1 levels. Moreover, there was not statistical association between toxicities (hematologic and non-hematologic) and hENT1 levels (Table 2). Finally, we did not find any statistically significant correlation between hENT1 protein staining and tumor response to gemcitabine based regimens $(p=0.735)$ (Table 2$)$. On the other hand, hENT1 protein staining was significantly associated with TTP in the univariate analysis. There was an improvement in TTP for positive hENT1 staining patients (HR:
2.902; 95\% CI, 1.053-7.996; $\mathrm{p}=0.03)$. Specifically, the median TTP in positive hENT1 staining patients was 6.33 months, as compared to only 2.83 months in those with low hENT1 expression $(\mathrm{p}=0.03)$ (Table 2) (Fig. 2). Furthermore, those patients with overexpression of hENT1 showed a longer OS when compared with patients with low hENT1 levels, but this increase did not reach the statistical significance (HR: 2.08; 95\% CI, 0.809-5.345; p=0.12). Specifically, the median OS in positive hENT1 staining patients was 14.0 months, as compared to only 7.0 months in those with low hENT1 expression $(\mathrm{p}=0.12)$ (Table 2) (Fig. 3). Then, we correlated the hENT1 levels of the 18 patients treated with gemcitabine at fixed dose rate and capecitabine with TTP and OS. In this specific subgroup of patients hENT1 protein staining was significantly associated also with OS (18.8 months in hENT1 positive vs 6.5 months in hENT1 negative patients) (HR: 8.024; 95\% CI, 1.36-47.30; $\mathrm{p}=0.02$ ).

In conclusion, besides hENT1 staining, none of the other parameters considered produced a separation in the survival curves after gemcitabine initiation.

\section{DISCUSSION AND CONCLUSION}

BTCs are invasive carcinomas deriving from the epithelial lining of the gallbladder and bile ducts. Patients with unresectable or metastatic BTC have a poor prognosis with a median OS <1 year [16].

Recently, a pooled analysis was conducted between 1999 and 2006 on 104 chemotherapy trials involving 1,368 BTC patients. This analysis underlined a biologic difference between colangiocarcinoma and gallbladder carcinoma and described gemcitabine as the most active agent [17]. Preclinical and translational data suggest that nucleoside transporters, in particular hENT1, play an important role in pre-

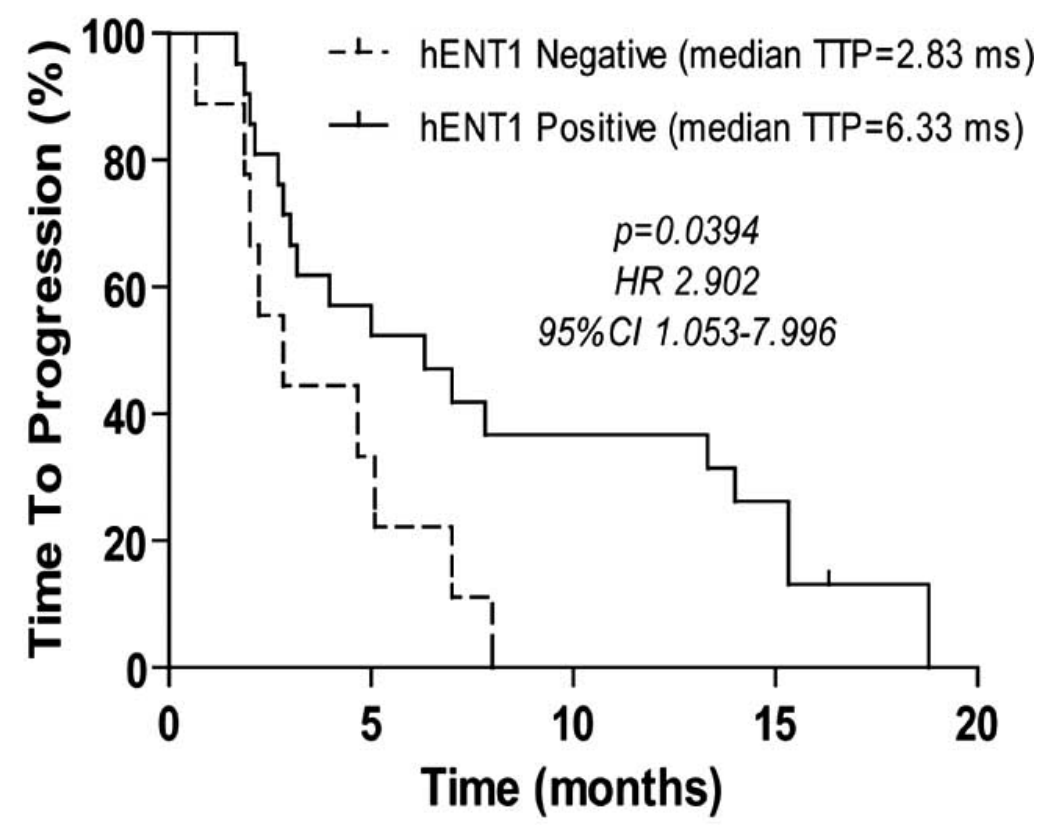

Fig. (2). Time To Progression in BTC patients receiving gemcitabine-based therapy according to hENT1 expression levels by tumor cells. The median time to progression period for positive and negative hENT1 staining patients was 6.33 months and 2.83 months, respectively ( $\mathrm{p}=0.0394)$. TTP: time to progression; ms: months. 


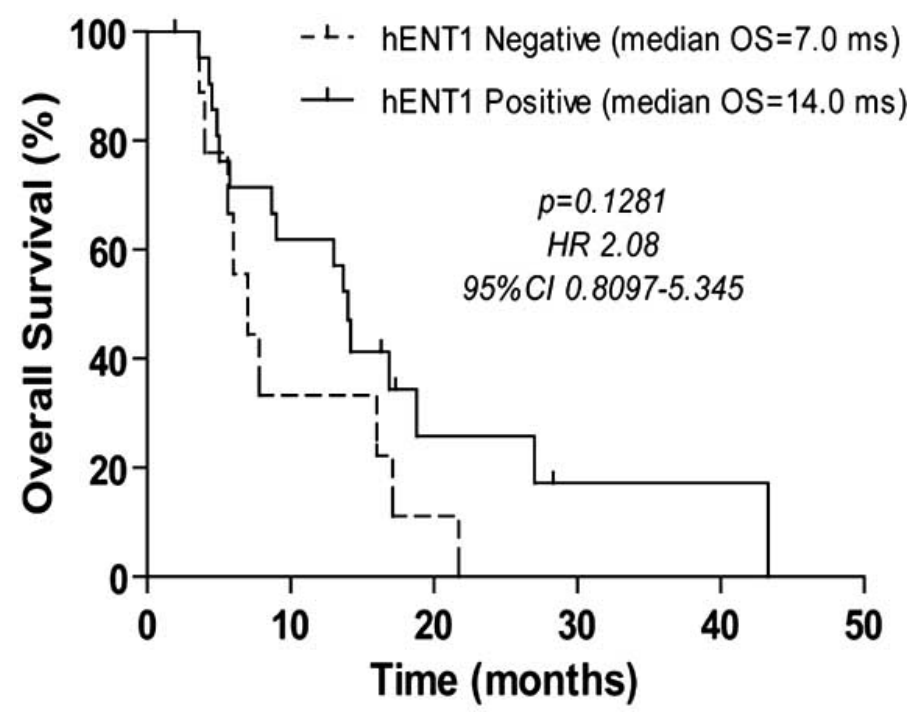

Fig. (3). Overall Survival in BTC patients receiving gemcitabine-based therapy according to hENT1 expression levels by tumor cells. The median time to progression period for positive and negative hENT1 staining patients was 14.0 months and 7.0 months, respectively $(\mathrm{p}=0.128)$. OS: overall survival; ms: months.

dicting clinical outcomes after gemcitabine chemotherapy for several types of cancer $[9,11,18]$.

In many clinical trials conducted in BTC patients, a significant but modest efficacy of classical cytotoxic chemotherapy has been achieved. Literature data suggest that gemcitabine and gemcitabine-based platinum regimens offer a real but slight advantage over other regimens [2] ${ }^{1}$. Only a minority of patients really benefits from chemotherapy in term of response rate and mostly in term of survival. For this reason, it is now necessary to find molecular or genetic factors predictive of response to chemotherapy, to better select patients and avoid both useless toxicities and increased health care costs.

This represents the first original study demonstrating how a molecular marker such as the IHC staining for the hENT1 protein may carry predictive information in patients with metastatic BTC. hENT1 protein expression in our patients has not demonstrated prognostic significance. In the subpopulation treated with gemcitabine and capecitabine hENT1 correlated also with OS. hENT1 is probably able to mediate the intracellular transport not only of gemcitabine but also of fluoropyrimidines, such as capecitabine [19]. From this point of view we can suppose that the correlation between hENT1 and OS, in this particular subgroup of patients, is linked to the potential impact of this chemotherapeutic regimen on metastatic BTC patients' outcome.

Among the BTCs, there are several differences related to disease course, sensitivity to chemotherapy, and molecular profiles on the basis of the site of primary tumor: intrahepatic, distal biliary tree, or gallbladder. Thus, while these different types of BTCs have traditionally been included together in clinical trials, it would be appropriate to separate these diseases in future studies [20].

Evidence of heterogeneity in chemotherapy response, genetics, and OS among the different BTCs on the basis of origin suggests that future trials evaluating the predictive role of hENT should either stratify these entities or study one specific entity in a specific trial. Given the relatively small incidence of these cancers, such a trial design will be a great challenge and will require a multicenter design to adequately include a statistically representative number of each subtype of BTC [16].

Understanding completely how tumor genetics predicts drug sensitivity is a key area to focus on in future trials. Whether these assays provide sufficient predictive information to guide treatment decisions requires prospective evaluation in randomized clinical trials. However, the present study highlights the potential role of hENT1 in predicting gemcitabine activity and efficacy and in selecting the subpopulation of BTC patients who could benefit from gemcitabine based regimes, avoiding futile treatments in potential nonresponder patients.

\section{ACKNOWLEDGEMENTS}

We thank Lottomatica for financial support.

\section{REFERENCES}

[1] Scheithauer, W. Review of gemcitabine in biliary tract carcinoma. Semin. Oncol. 2002, 29(6 Suppl 20), 40-45.

[2] Dingle, B.H.; Rumble, R.B.; Brouwers, M.C. The role of gemcitabine in the treatment of cholangiocarcinoma and gallbladder cancer: a systematic review. Can. J. Gastroenterol. 2005, 19(12), 711716.

[3] Pasetto, L.M.; D'Andrea, M.R.; Falci, C.; Monfardini, S. Gemcitabine in advanced biliary tract cancers. Crit. Rev. Oncol. Hematol. 2007, 61(3), 230-242.

[4] Baldwin, S.A.; Mackey, J.R.; Cass, C.E.; Young, J.D. Nucleoside transporters: molecular biology and implications for therapeutic development. Mol. Med. Today 1999, 5(5), 216-224.

[5] Mackey, J.R.; Mani, R.S.; Selner, M.; Mowles. D.; Young, J.D.; Belt, J.A.; Crawford, C.R.; Cass, C.E. Functional nucleoside transporters are required for gemcitabine influx and manifestation of toxicity in cancer cell lines. Cancer Res. 1998, 58(19), 4349-4357.

[6] Chow, L.; Lai, R.; Dabbagh, L.; Belch, A.; Young, J.D.; Cass, C.E.; Mackey, J.R. Analysis of human equilibrative nucleoside 
transporter 1 (hENT1) protein in non-Hodgkin's lymphoma by immunohistochemistry. Mod. Pathol. 2005, 18(4), 558-564. [7] Damaraju, V.L.; Elwi, A.N.; Hunter, C.; Carpenter, P.; Santos, C.;
Barron, G.M.; Sun, X.; Baldwin, S.A.; Young, J.D.; Mackey, J.R.; Sawyer, M.B.; Cass, C.E. Localization of broadly selective equilibrative and concentrative nucleoside transporters, hENT1 and hCNT3, in human kidney. Am. J. Physiol. Renal. Physiol. 2007, 293(1), F200-211.

[8] Mackey, J.R.; Jennings, L.L.; Clarke, M.L.; Santos, C.L.; Dabbagh, L.; Vsianska, M.; Koski, S.L.; Coupland, R.W.; Baldwin, S.A.; Young, J.D.; Cass, C.E. Immunohistochemical variation of human equilibrative nucleoside transporter 1 protein in primary breast cancers. Clin. Cancer Res. 2002, 8(1), 110-116.

[9] Seve, P.; Mackey, J.R.; Isaac, S.; Trédan, O.; Souquet, P.J.; Pérol, M.; Cass, C.; Dumontet, C. cN-II expression predicts survival in patients receiving gemcitabine for advanced non-small cell lung cancer. Lung. Cancer 2005, 49(3), 363-370.

[10] Spratlin, J.; Sangha, R.; Glubrecht, D.; Dabbagh, L.; Young, J.D.; Dumontet C.; Cass, C.; Lai, R.; Mackey, J.R. The absence of human equilibrative nucleoside transporter 1 is associated with reduced survival in patients with gemcitabine-treated pancreas adenocarcinoma. Clin. Cancer Res. 2004, 10(20), 6956-6961.

[11] Farrell, J.J.; Elsaleh, H.; Garcia, M.; Lai, R.; Ammar, A.; Regine, W.F.; Abrams, R.; Benson, A.B.; Macdonald, J.; Cass, C.E.; Dicker, A.P.; Mackey, J.R. Human equilibrative nucleoside transporter 1 levels predict response to gemcitabine in patients with pancreatic cancer. Gastroenterology 2009, 136(1), 187-195.

[12] Mori, R.; Ishikawa, T.; Ichikawa, Y.; Taniguchi, K.; Matsuyama, R.; Ueda, M.; Fujii, Y.; Endo, I.; Togo, S.; Danenberg, P.V.; Shimada, H. Human equilibrative nucleoside transporter 1 is associated with the chemosensitivity of gemcitabine in human pancreatic adenocarcinoma and biliary tract carcinoma cells. Oncol. Rep. 2007, 17(5), 1201-1205.

[13] Santini, D.; Perrone, G.; Vincenzi B.; Lai, R.; Cass, C.; Alloni, R.; Rabitti, C.; Antinori, A.; Vecchio, F.; Morini, S.; Magistrelli, P.; Coppola, R.; Mackey, J.R.; Tonini, G. Human equilibrative nucleoside transporter $1(\mathrm{hENT} 1)$ protein is associated with short survival in resected ampullary cancer. Ann. Oncol. 2008, 19(4), 724-728.

[14] Eckel, F.; Jelic, S. Biliary cancer: ESMO clinical recommendation for diagnosis, treatment and follow-up. Ann. Oncol. 2009, 20 (Suppl 4), 46-48.

[15] Rosai, J. Rosai and Ackerman's surgical pathology, 9th ed; Mosby: Edinburgh, 2004, 2953-2954.

[16] Hezel, A.F.; Zhu, A.X. Systemic therapy for biliary tract cancers. Oncologist 2008, 13(4), 415-423.

[17] Eckel, F.; Schmid, R.M. Chemotherapy in advanced biliary tract carcinoma: a pooled analysis of clinical trials. Br. J. Cancer 2007, 96(6), 896-902.

[18] Marechal, R.; Mackey, J.R.; Lai, R.; Demetter, P.; Peeters, M.; Polus, M.; Cass, C.E.; Young, J.; Salmon, I.; Devière, J.; Van Laethem, J.L. Human equilibrative nucleoside transporter 1 and human concentrative nucleoside transporter 3 predict survival after adjuvant gemcitabine therapy in resected pancreatic adenocarcinoma. Clin. Cancer Res. 2009, 15(8), 2913-2919.

[19] Molina-Arcas, M.; Moreno-Bueno, G.; Cano-Soldado, P.; Hernández-Vargas, H.; Casado, F.J.; Palacios, J.; Pastor-Anglada, M. Human equilibrative nucleoside transporter-1 (hENT1) is required for the transcriptomic response of the nucleoside-derived drug 5'DFUR in breast cancer MCF7 cells. Biochem. Pharmacol. 2006 , 72(12), 1646-1656.

[20] Tonini, G.; Fratto, M.E.; Vincenzi, B.; Santini, D. Classification of biliary tract cancer (BTC): evaluation of all entities. Ann. Oncol. 2009, 20(6), 1148-1149. 